\title{
Burden of malaria in children under five and caregivers' health-seeking behaviour for malaria-related symptoms in artisanal mining communities in Ghana
}

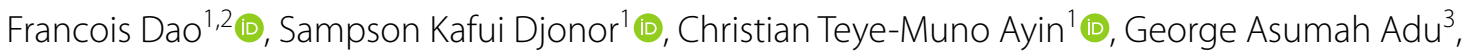
Bismark Sarfo ${ }^{1}$, , Pricillia Nortey $^{1}$, Kwadwo Owusu Akuffo ${ }^{4}$ and Anthony Danso-Appiah ${ }^{1,5^{*}}$ (1)

\begin{abstract}
Background: Artisanal mining creates enabling breeding ground for the vector of malaria parasites. There is paucity of data on the effects of artisanal mining on malaria. This study assessed burden of malaria and caregivers' healthseeking behaviour for children under five in artisanal mining communities in East Akim District in Ghana.

Methods: A cross-sectional study involving caregivers and their children under five was conducted in three artisanal mining communities in the East Akim District in Ghana. Caregivers were interviewed using a structured questionnaire. Finger prick blood samples were collected and analysed for haemoglobin concentration using a rapid diagnostic test, and thick and thin blood smears were analysed to confirm the presence of malaria parasites.

Results: Of the 372 children under 5 years included in the study, 197 (53.1\%) were male, with a mean age ( \pm SD) of $23.0 \pm 12.7$ months. The proportion of children with malaria (Plasmodium falciparum and P. malariae) was $98.1 \%$ and $1.9 \%$, respectively, whilst the proportion with anaemia $(\mathrm{Hb}<11.0 \mathrm{~g} / \mathrm{dl})$ was $39.5 \%(n=147)$. Almost all caregivers were female $(98.9 \%)$, and $28.6 \%(n=106)$ did not have access to any malaria control information. Caregivers associated malaria infection with mosquito bites $(68.3 \%, n=254)$ and poor sanitation $(21.2 \%, n=79)$. Malaria in children under five was significantly associated with anaemia (OR 11.07, 95\% Cl 6.59-18.68, $n=111 / 160,69.4 \% ; P<0.0001$ ), residing close to stagnant water $(\leq 25 \mathrm{~m}$ ) from an artisanal mining site (AOR 2.91,95\% Cl 1.47-5.76, $P=0.002$ ) and caregiver age younger than 30 years (OR $0.44,95 \% \mathrm{Cl} 0.208-0.917, n=162,43.55 \%, P=0.001$ ).

Conclusions: There is a high burden of malaria and anaemia among children under five in artisanal mining communities of the East Akim District, and far higher than in non-artisanal mining sites. Interventions are needed to effectively regulate mining activities in these communities, and strengthen malaria control and health education campaigns to curtail the high malaria burden and improve health-seeking behaviour.
\end{abstract}

Keywords: Prevalence, Malaria transmission, Vector control, Galamsey activities, Anaemia, Plasmodium falciparum, Childhood malaria, Caregivers'behaviour, East Akim, Ghana

*Correspondence: tdappiah@yahoo.co.uk

${ }^{1}$ Department of Epidemiology and Disease Control, School of Public Health, University of Ghana, Legon, Ghana

Full list of author information is available at the end of the article

\section{Background}

After decades of control efforts, malaria still poses a serious public health threat, with 229 million estimated reported cases in 2019 [1] and 405,000 attributable deaths, of which two-thirds $(272,000)$ occurred in 
children under 5 years of age [2]. Deaths from malaria are caused mainly by the acute form of the disease, but much more is due to subtle effects, including anaemia. Anaemia remains a major complication and risk of death from malaria. In 2018, it was estimated that up to $79 \%$ of children under five in high-burden areas in Africa who were diagnosed with malaria had anaemia [3]. Malaria is the number one cause of morbidity, accounting for about $38 \%$ of all outpatient illnesses, and about $31 \%$ of all deaths in children under five [4], with almost all cases (97\%) caused by Plasmodium falciparum [5]. According to the World Health Organization (WHO), the African Region accounted for $94 \%$ of all malaria deaths in 2018 , and despite the relatively lower number of 180,000 deaths in that year, the region was still responsible for $85 \%$ of the deaths recorded [2].

A systematic review assessing patterns of malaria variation by age with respect to severity, transmission intensity and seasonality in sub-Saharan Africa found clinical malaria burden to be higher in younger age groups. Hospital admissions were also higher among younger children, with higher levels of mortality among infants [6].

In Ghana, malaria accounted for 10.4 million of outpatient department (OPD) visits in 2016 and was responsible for a case fatality rate of 0.32 among children under five [7]. That same year (2016), the East Akim District of the Eastern Region of Ghana, which is well known for its mining activities, including artisanal mining, recorded prevalence of $34.1 \%$ with the malaria rapid diagnostic test (m-RDT) among children aged 6 to 59 months, the highest in the region [8]. Several studies have sought to link the activities of artisanal mining of gold with the increased prevalence of malaria [9-11]. Deforestation, unrehabilitated mining pits containing stagnant water bodies and other mining-related activities are known to promote the proliferation of the female anopheles mosquitoes, the vector for the Plasmodium parasite responsible for most of the malaria cases in Ghana. Lack of adequate housing, with unsealed windows and doors allowing free entry of mosquitoes, and living less than $25 \mathrm{~m}$ from a stagnant water body have also been found to be factors associated with increased exposure to malaria risk $[11,12]$.

However, there is a paucity of data on the burden of malaria in children under five in the East Akim District. Furthermore, the extent to which ongoing artisanal mining is impacting the burden of malaria is largely unknown. Therefore, this study sought to assess the burden of malaria in children under five as well as caregivers' knowledge and healthcare-seeking behaviour for malariarelated symptoms in their children in an artisanal mining community in Ghana. Findings from this study could be utilized in planning future malaria control activities in similar settings.

\section{Methods \\ Type of study design and study area}

The study used a cross-sectional design and employed quantitative methods. It was conducted in three localities (Kyebi, Adadientem and Ahwenease) of East Akim District, an artisanal mining area in the Eastern Region of Ghana (Fig. 1). The East Akim Municipal Assembly has a total area of about $725 \mathrm{~km}^{2}$. According to the 2010 Population and Housing Census [13], the population of the Akim Municipality was estimated at 167,896 inhabitants. The municipality is located in the western semiequatorial zone, which is characterized by two main rainfall seasons, May-June and September-October, with average annual rainfall of between 125 and $175 \mathrm{~mm}$. Temperatures are relatively uniform, ranging from $26{ }^{\circ} \mathrm{C}$ in August to $30{ }^{\circ} \mathrm{C}$ in March. The relative humidity is generally high year-round, ranging between 70 and $80 \%$. The municipality is in the semi-deciduous rainforest with a forest reserve covering about $108.8 \mathrm{~km}^{2}$, representing approximately $15 \%$ of the total area. The district is heavily endowed with mineral deposits-gold, diamond, bauxite, etc. But the mining sector is dominated by the activities of artisanal miners, which exist in almost every community of the district. The district is made up of 27 communities, of which 21 are rural.

The municipality is divided into seven health administrative sub-municipalities: Amanfrom, Apedwa, Asafo, Asiakwa, Bunso, Kibi and Maase. There is one municipal hospital, one polyclinic, four health centres, 15 community-based health planning service (CHPS) compounds and 29 demarcated CHPS zones. Malaria, upper respiratory infections, rheumatism and joint pain, intestinal worm infestation and acute respiratory tract infection are the typical cases reported to health facilities. With the introduction of the National Health Insurance Scheme (NHIS) in 2006, services and care rendered by these facilities are covered by the scheme, although the roughly $30 \%$ of patients not registered with the NHIS would have to pay out-of-pocket for services received.

\section{Study population}

The study population comprised children under 5 years of age and their caregivers who had lived in the East Akim artisanal mining communities for at least a month. One month was chosen to reflect the incubation period of the Plasmodium parasites [12]. Children who were eligible to be included but whose caregivers failed to give consent to the study protocols were excluded. Each child was linked directly to their caregiver in the study. 


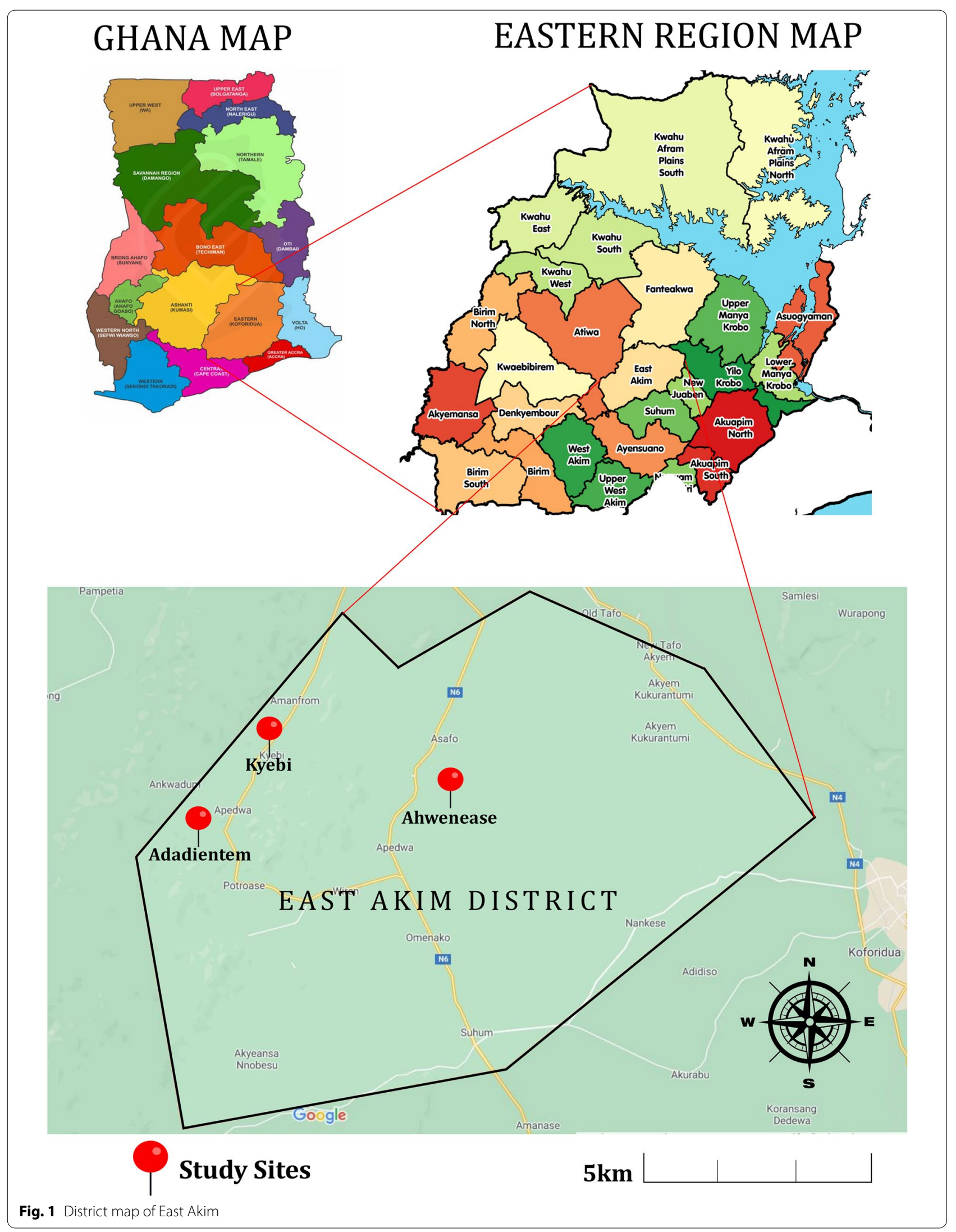




\section{Sample size determination and sampling}

According to the Ghana Malaria Indicator Survey 2016 [8], the Eastern Region recorded the highest malaria cases by microscopy, with $31.1 \%$ in children aged $6-59$ months (under 5 years). Using this proportion, the minimum sample size was derived using Cochran's formula:

$$
N=\frac{z^{2}(p \times q)}{d^{2}},
$$

where $N=$ sample size; $p=$ malaria proportion by microscopy in the Eastern region (31.1\%); $q=1-p ; z=$ the critical probability value for a confidence level of $95 \%$ (1.96) and $d=5 \%$ margin of error $(0.05)$.

This gives $N=\frac{(1.96)^{2}(0.311 \times 0.689)}{(0.05)^{2}}=329.27$.

With an assumed estimate of $10 \%$ non-response rate, a minimum total sample size of 362 children (one child per caregiver) was obtained for the entire study.

The East Akim District has nine artisanal mining communities with different population sizes. Three communities were randomly selected for this study, namely Adadientem, Kyebi and Ahwenease. The communities were sampled proportionately based on the community size and translated into a ratio of 30:40:30, respectively. Figure 2 summarizes the recruitment process.

A total of 112 children under 5 years from Adadientem, 148 from Kyebi and 112 from Ahwenease, together with their caregivers, were sampled for the study. With the knowledge of estimated total number of houses in these communities, a proportionate sampling method was used to select the number of houses included in the study. The researcher tossed a pen, and where the tip of the pen pointed marked the direction of movement. The first house in that direction was chosen, and the third number from that house was selected in that order. One child under 5 years was selected in each house; in houses with more than one qualified participant, a simple random sampling was used to select one of them. Also, an adjacent house with a qualified participant was used to replace houses without qualified participants or with a participant who declined to participate in the study. The distance between a house and a mining site with stagnant water was determined using a Global Positioning System receiver (Garmin eTrex 20).

\section{Blood sample collection technique}

Finger prick blood samples were collected from children under five to measure the haemoglobin concentration using the $\mathrm{HemoCue}^{\circledR} \mathrm{Hb} 201+$ system and to prepare thick and thin smears for observation under a light microscope (Olympus CX22 binocular microscope, $100 \times 100)$ for identification of asexual malaria parasites after staining with $10 \%$ Giemsa. According to standard WHO procedures, haemoglobin concentrations and blood smear results were then reported as soon as possible after observation by certified microscopists. Malaria parasitaemia was defined as the presence of an asexual form of any Plasmodium species detected by microscopy. Asymptomatic malaria was defined as the presence of an asexual form of any microscopically detected plasmodial species with the absence of symptomatic malaria or history of fever over the last 2 weeks. Participants were also examined with the SD BIOLINE Malaria Ag P.f rapid diagnostic test (RDT; HRP2/pLDH). Participants who tested positive with RDT or microscopy were referred for malaria treatment at the nearest health centre.

\section{Questionnaire and data collection technique}

The questionnaire used in this study had four main sections. The first section collected information on children's and caregivers' demographic characteristics; the second, data from caregivers on their knowledge of malaria transmission, signs and symptoms; the third, information on their child's health and history of malaria signs and symptoms; and the last on caregivers' healthseeking behaviour.

\section{Quality assurance and quality control}

The survey was conducted by experienced research assistants who were trained in the study protocols. All samples taken were marked with a participant identification number and recorded in a register. Two certified microscopists, who were blinded to the RDT results, carried out the microscopy for malaria parasites. A third microscopist was consulted in the case of any divergence greater than or equal to $50 \%$. The HemoCue system and microscopes were calibrated and verified prior to use in the study. The RDT kits were approved by the Food and Drugs Authority (FDA), as recommended by the Ghana National Malaria Control Programme (NMCP). The questionnaire was completed and verified by the data collection team for accuracy, consistency and completeness.

\section{Data processing and analysis}

A mobile app (REDCap ${ }^{\circledR}$ software) was downloaded as a Microsoft Excel file and used for the data collection. Stata version 15.0 was used for the statistical analysis. The level of malaria parasitaemia was determined by counting the number of asexual parasites in blood film against 200 leucocytes counted alongside these parasites, and by assuming 8000 leukocytes $/ \mathrm{ml}$; the ratio obtained was then multiplied by the leucocyte count to determine the parasite density per microlitre of blood for each participant with a positive malaria test. Haemoglobin values 


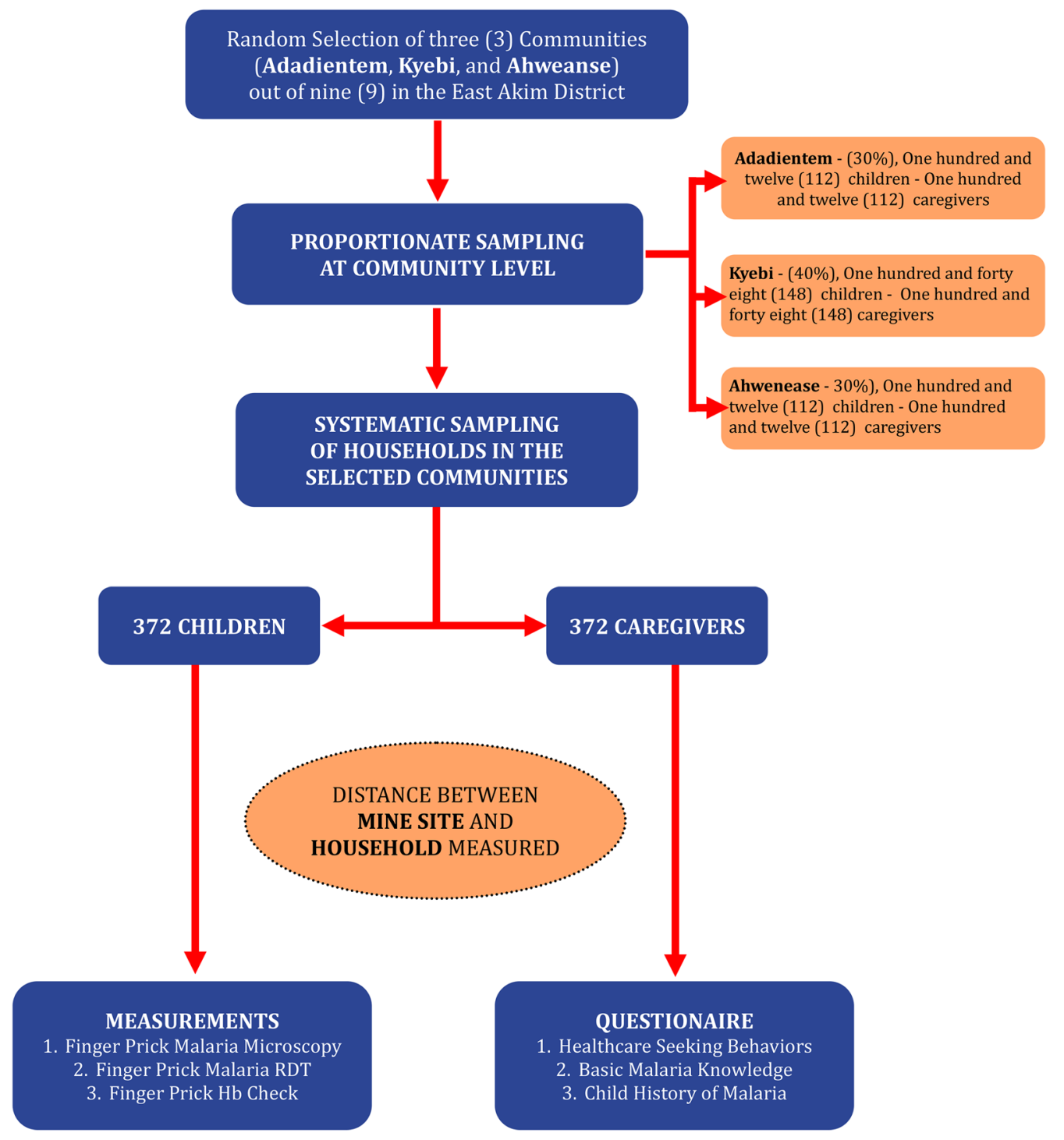

Fig. 2 Flow chart illustrating the recruitment procedure

less than $11.0 \mathrm{~g} / \mathrm{dl}$ were classified as anaemia present, and values above $11.0 \mathrm{~g} / \mathrm{dl}$ described as no anaemia. The categorical variables were presented as frequencies and percentages. Univariate logistic regression was run for all the variables, and those found to be statistically significant at $P<0.05$ were further analysed using multivariate logistic regression. All estimates were presented with their $95 \%$ CIs.

\section{Results}

Socio-demographic characteristics of children under five Though we set out to recruit a minimum of 362 children, the study sampled a total of 372 children, with mean age of 23 months (SD 12.7 months). Two thirds (66.9\%) of the children were aged $\leq 11$ months and 197 (53.1\%) were male.

\section{History of presence of malaria signs/symptoms and intensity among the children}

Two hundred and seventy-five (275) children reported an episode of fever more than 1 month prior to the study. The fever was of moderate intensity in 208 (75.6\%) children, with 197 (71.6\%) of the children who had fever receiving medical care from the hospital or clinic. Among those not treated in either the hospital or clinic, 34 (12.4\%) caregivers believed that self-medication was a better option. Regarding the history of the 
presence of malaria signs and symptoms and intensity, 19 (5.1\%), 11 (2.9\%) and 20 (5.4\%) children experienced convulsions, pallor and somnolence, respectively. The number of reported symptoms increased according to the duration of the disease: between 2 weeks and 1 month for convulsions (16; 84.2\%), pallor (6; 54.6\%) and somnolence $(16 ; 80.0 \%)$. Symptom intensity was more severe with convulsions $(17 ; 89.5 \%)$ and somnolence $(17 ; 85.0 \%)$ than with pallor $(5 ; 45.5 \%)$. A significant proportion of cases of convulsions, pallor and somnolence were reported to have been treated in a health facility $[16(84.2 \%), 8(72.7 \%)$ and 17 (85.0\%), respectively] (Additional file 1: Table S1).

\section{Children's medical history}

In this study, $134(36.0 \%)$ children were reportedly experiencing a malaria-related sign or symptom on the day of the survey. Of the 156 (41.9\%) children who were reported to have been sick with malaria in the past month, treatment was sought by caregivers for 143 (91.7\%), mostly in a health facility (109; 76.2\%). Twenty-four (16.8\%) visited a pharmacy, one $(0.7 \%)$ caregiver engaged in self-medication, and two (1.4\%) sought care from a faith healer. Sixty (37.0\%) children were asymptomatic carriers of malaria.

\section{Socio-demographic characteristics of caregivers}

A total of 372 caregivers were interviewed, of whom the majority $(368 ; 98.9 \%)$ were female, with a mean age of 26.5 years (SD 5.4), and a majority were married (67.1\%). Most caregivers had a low level of education, with primary or junior secondary school (JSS)/senior high school (SHS) representing 75.8\%. One hundred and fifty-eight $(42.5 \%)$ were self-employed, and the vast majority $(365 ; 98.1 \%)$ were of the Christian faith (Table 1).

\section{Socio-environmental factors}

The majority of caregivers $(349 ; 93.8 \%)$ said that they had well-sealed windows and doors, with 304 (81.7\%) having well-screened windows. Two hundred and seventy-three respondents (42.5\%) had a place of residence close to a mine site (within $25 \mathrm{~m}$ from the mine site), and 198 (53.2\%) had stagnant and open wells created from mining activities close to their place of residence.

\section{Caregivers' health-seeking behaviour}

Regarding caregivers' health-seeking behaviour, 234 (62.9\%) caregivers sought medical care for their children at health facilities. Forty-seven (12.6\%) engaged in other practices such as self-medication or visiting a spiritualist
Table 1 Socio-demographic characteristics of caregivers

\begin{tabular}{|c|c|c|}
\hline Characteristic & $\begin{array}{l}\text { Frequency } \\
(n=372)\end{array}$ & Percentage \\
\hline \multicolumn{3}{|l|}{ Sex of caregiver } \\
\hline Male & 4 & 1.1 \\
\hline Female & 368 & 98.9 \\
\hline \multicolumn{3}{|c|}{ Educational status of caregiver } \\
\hline No formal education & 24 & 6.5 \\
\hline Primary/JHS/SHS & 282 & 75.8 \\
\hline Secondary & 58 & 15.6 \\
\hline Tertiary (university) & 8 & 2.1 \\
\hline \multicolumn{3}{|l|}{ Occupation (caregiver) } \\
\hline Civil servants & 12 & 3.2 \\
\hline Self-employed & 158 & 42.5 \\
\hline Trading & 87 & 23.4 \\
\hline Unemployed & 91 & 24.5 \\
\hline Others & 24 & 6.4 \\
\hline \multicolumn{3}{|l|}{ Religion (caregiver) } \\
\hline Christianity & 365 & 98.1 \\
\hline Islam & 4 & 1.1 \\
\hline Traditional (fetishism) & 2 & 0.5 \\
\hline Other & 1 & 0.3 \\
\hline \multicolumn{3}{|l|}{ Marital status (caregiver) } \\
\hline Single & 113 & 30.5 \\
\hline Married & 249 & 67.1 \\
\hline Divorced & 3 & 0.8 \\
\hline Separated & 6 & 1.6 \\
\hline
\end{tabular}

JHS junior high school, SHS senior high school

or herbalist. Most (79.4\%) caregivers who resorted to other forms of treatment rather than seeking care from health facilities said they practiced self-medication. Almost 9\% (8.8\%) of those who sought other forms of care said they did so because of high healthcare cost.

\section{Caregivers' awareness and knowledge of malaria}

The results showed that 106 (28.6\%) caregivers did not have access to any malaria control information. Thirtyfour $(9.1 \%)$ accessed malaria information at least six times per month. One hundred and twenty-seven (40.8\%) of the respondents accessed malaria information through the radio, whilst 102 (40.8\%) accessed through television. Few respondents accessed malaria information from health workers and friends. Approximately 350 (94\%) caregivers knew about malaria and were aware of how it is transmitted, with most associating it with mosquito bites $(254 ; 68.3 \%)$ and poor sanitation $(79 ; 21.2 \%)$. 


\section{Association between malaria and anaemia among the children}

Two hundred and two (54.3\%) of the children tested positive for malaria by malaria RDT, whilst 160 (43.0\%) of the slides showed the presence of malaria parasite by microscopy, indicating an $11 \%$ difference between the two diagnostic criteria. Plasmodium falciparum constituted the majority of the infections (98.1\%). One child had mixed infections of $P$. malariae and P. falciparum. The mean $\mathrm{Hb}$ of the children was found to be 11.4 (95\% CI 11.2-11.5) $\mathrm{g} / \mathrm{dl}$, with a minimum $\mathrm{Hb}$ reading of $7.2 \mathrm{~g} / \mathrm{dl}$ and maximum of $15.2 \mathrm{~g} / \mathrm{dl}$. One hundred and forty-seven (39.5\%) of the children had anaemia $(\mathrm{Hb}<11.0 \mathrm{~g} / \mathrm{dl})$ (Additional file 1: Table S2).

Using the thick/thin blood smear, which is the gold standard for malaria diagnosis, the prevalence of malariaassociated anaemia in children under five was found to be $69.4 \%$. Anaemia was found to be associated with malaria in children under five (Table 2). Among all 147 children who had anaemia, $136(92.5 \%)$ tested positive for malaria by RDT (OR 29.8, 95\% CI 15.1-58.7, P<0.0001) whilst 111 (75.5\%) also tested positive for malaria from blood smear (OR 11.07, 95\% CI 6.8-18.1, P<0.0001).

From bivariate analysis of caregivers' socio-demographics and malaria infection, significant associations with infection were found for both child and caregiver age (Additional file 1: Table S2). Caregivers who were 30 years old and older were less likely to have their children infected with malaria compared to those younger than 30 (OR $0.4,95 \%$ CI $0.2-0.9, P=0.017$ ). However, caregivers' marital status $(P=0.753)$, employment $(P=0.928)$ and formal educational status $(P=0.753)$ and the sex of the child $(P=0.206)$ were all found not to be significant.

\section{Caregiver's knowledge of malaria and incidence of malaria in children under five from bivariate analysis}

The bivariate analysis revealed that knowledge of malaria control information among caregivers was protective against malaria infections in children (OR 0.7, 95\% CI $0.4-1.3, P=0.207)$. However, the difference between good and poor knowledge of malaria control information among caregivers was not statistically significant in influencing malaria infections in children under five.

\section{Bivariate analysis of socio-environmental factors and malaria infection}

The study found a significant association between place of residence close to mine sites and malaria infection. Children of caregivers who lived less than or equal to $25 \mathrm{~m}$ from mine sites were 4.63 times as likely to be infected with malaria as those who lived more than $25 \mathrm{~m}$
Table 2 Association between anaemia and malaria among children under 5 years living in artisanal mining communities in Ghana

\begin{tabular}{|c|c|c|c|}
\hline \multirow[t]{2}{*}{ Variables } & \multicolumn{2}{|c|}{ Anaemia status } & \multirow[t]{2}{*}{ Crude OR (95\% Cl) } \\
\hline & Absent (\%) & Present (\%) & \\
\hline \multicolumn{4}{|l|}{ Malaria (RDT results) } \\
\hline Negative & $159(70.7)$ & $11(7.5)$ & 0.07 \\
\hline Positive & $66(29.3)$ & $136(92.5)$ & $29.8(15.1-58.7)$ \\
\hline \multicolumn{4}{|l|}{ Malaria (smear results) } \\
\hline No MPs seen & $176(78.2)$ & $36(24.5)$ & 0.20 \\
\hline MPs seen & $49(21.8)$ & $111(75.5)$ & $11.1(6.8-18.1)$ \\
\hline \multicolumn{4}{|l|}{ Plasmodium infection } \\
\hline Negative & $176(78.2)$ & $36(24.5)$ & 0.20 \\
\hline $\begin{array}{l}\text { Mono-infection (asexual } \\
\text { form) }\end{array}$ & $49(21.8)$ & $110(74.8)$ & $11.0(6.7-18.0)$ \\
\hline $\begin{array}{l}\text { Mixed infection (asexual } \\
\text { form) }\end{array}$ & $0(0.0)$ & $1(0.3)$ & - \\
\hline
\end{tabular}

$R D T$ rapid diagnostic test, $C l$ confidence interval, OR odds ratio, MPs malaria parasites

from mine sites (OR 4.6, 95\% CI 2.6-8.5, $P=0.001$ ). Furthermore, children whose caregivers lived $25 \mathrm{~m}$ or less from stagnant clean water wells due to mining were 2.67 times as likely to be infected with malaria as those who did not live close to mine sites (OR 2.8, 95\% CI 1.7-4.2, $P=0.001)$. However, the presence or absence of window door screens did not show any statistical significance with regard to malaria infection $(P=0.916)$.

\section{Multiple logistic regression analysis}

All variables described in Additional file 1: Table S2 were examined for malaria infections in multivariate analysis. These variables included caregiver and child age, caregiver marital status, employment, education, frequency with which child contracted malaria, closeness $(\leq 25 \mathrm{~m})$ of residence to mining-created stagnant wells, closeness of residence to mining site, and caregiver's access to malaria control information. These were variables that were found significant at the bivariate analysis level or considered to have a theoretically significant influence on malaria infection in artisanal and small-scale mining (ASM) communities. Only the frequency with which a child contracted malaria and the closeness $(\leq 25 \mathrm{~m})$ of the residence to mining-created stagnant wells remained significant in the multivariate model. Caregivers whose homes were close $(\leq 25 \mathrm{~m})$ to stagnant water wells due to mining were 2.9 times as likely to have their child infected with malaria as those who did not live close to stagnant clean water wells (OR 2.9, 95\% CI 1.5-5.8, $P=0.002)$. Children who often contracted malaria were also twice as likely to have malaria infections as those 
who did not often contract malaria (OR 2.0, 95\% CI 1.1$3.8, P=0.023)$. No other factors were found to be significantly associated with malaria infections in ASM sites in the multivariate analysis.

\section{Discussion}

\section{Malaria among children under 5 years in ASM sites} in Ghana

The study assessed the malaria burden among children under five in the artisanal mining communities of East Akim and caregivers' knowledge in relation to malaria. The study found $54.3 \%$ cases of malaria using RDT and $43 \%$ with microscopy. Malaria was a good predictor of anaemia, with malaria-associated anaemia found in $69.4 \%$ of cases. Caregivers seeking medical care from health facilities accounted for $62.9 \%$, of whom $94 \%$ had knowledge of malaria and mode of transmission of the infection. Living in a residence less than $25 \mathrm{~m}$ from a stagnant clear water well due to artisanal mining increased the risk of malaria for children under five by threefold.

Several studies have sought to estimate the burden of malaria in children under five, usually with wide variations in prevalence. A survey by Yankson et al., which sought to assess the risk of malaria in children under five by analysing the 2016 Ghana Demographic Health Survey data, found malaria prevalence of $22.1 \%$ [14]. An intervention study in children under five in Lagos, Nigeria, showed malaria parasitaemia in $58.8 \%$ at baseline [15]. Another study in Uganda found malaria prevalence of $19.0 \%$ and a study in rural Malawi found $33.8 \%$ prevalence using a cross-sectional survey [16, 17]. Generally, high malaria prevalence in children under five has been associated with increasing child age, low educational status of mother, and coming from both a poor household and rural area $[14,16-18]$. In this study, at artisanal mining areas, malaria prevalence in children under five was relatively high (43\%) compared to previously cited studies. The high malaria prevalence rate in artisanal mining areas could be due to massive environmental changes that affect vector abundance and transmission behaviour [19]. ASM activity leaves stagnant dirty water that serves as a breeding ground for mosquitoes and promotes the spread of malaria. We also found that having frequent episodes of malaria was significantly associated with malaria infection in these artisanal mining communities. Despite our inability to identify a cause-and-effect relationship in this cross-sectional study, we strongly believe that ASM and its associated environmental conditions predispose children under 5 years to malaria infection, and are reasons why children in these study sites have frequent episodes of malaria. Until breeding grounds of mosquitoes left behind by ASM are identified and controlled, infection will continue. This explains why malaria could be frequent in these children.

\section{Malaria-associated anaemia among children under 5 years in ASM sites}

The prevalence of malaria-associated anaemia was $69.4 \%$ in this study. A child under five with a positive malaria RDT had 30-fold increased odds of anaemia compared to a similar child with negative malaria RDT. Likewise, having a positive malaria smear increased the odds of a child under five having anaemia by 11 -fold. Similar results have been reported in studies in low-resource mining settings of Okada in Nigeria, the Bonikro mining area in central Cote d'Ivoire, and Luangwa District in Zambia [20-22]. Erythrocyte lysis and phagocytosis and sequestration of parasitized red blood cells have been proposed as possible explanations for malarial anaemia, with recent data also indicating bone marrow suppression and ineffective erythropoiesis as possible pathways $[23,24]$. A large study in Ghana involving 2123 children conducted around the same period as the current study found anaemia and malaria prevalence of $35.6 \%$ and $20.3 \%$, respectively, in children under 5 years [25]. An earlier household survey of 7739 children under 5 years in 2014 also found $40 \%$ anaemia prevalence [26]. About 3 years later, where several conditions contributing to anaemia in the country should have been better, anaemia prevalence of over $60 \%$ would not have been expectedand is strongly associated with malaria in this study and owing to artisanal mining in these sites. Mining activities always leave behind environmentally unfavourable conditions such as abundant bushy areas and dug holes that accumulate dirty stagnant water that serves as breeding grounds for mosquitoes and the spread of malaria.

\section{Asymptomatic and Plasmodium species-specific malaria in ASM sites in Ghana}

This study saw about a third of asymptomatic carriers of malaria parasites among children under five. Similar findings have been reported in several studies, including artisanal gold mining settings in Guyana. Inadequate malaria treatment, which limits parasite densities without actually eliminating them, has been linked to clinically silent malaria. Asymptomatic carriers serve as reservoirs for malaria parasites, likely becoming a channel for intense malaria transmission when mosquitoes start breeding during the rainy season [19, 27-29].

About half of the children tested positive for malaria by RDT, whilst a slightly lower proportion (43.0\%) had positive slides for malaria in this study. Plasmodium falciparum accounted for the majority of malaria infections, consistent with the World Health Organization report, which indicates that $P$. falciparum remains the most 
common of the five Plasmodium species that are known to cause malaria disease among Africans [2]. Additionally, findings support the data from the Ghana Health Service (GHS), where malaria caused by $P$. falciparum species accounts for almost $97 \%$ of cases $[4,5]$.

\section{Effect of ASM on malaria infections in nearby communities} In the current study, among the factors studied that could influence the risk of malaria infection, children under five whose caregivers lived less than $25 \mathrm{~m}$ from an open stagnant clean water well as a result of mining had significantly increased risk of malaria infection. This finding is consistent with studies conducted in both Ghana and southern Ethiopia, which reported children under five living near mining areas being disproportionately exposed to mosquitoes bites [30,31]. Malaria and its associated anaemia can lead to significant morbidity and mortality. Several studies [14, 16-19] have pointed out how the high rate of malaria infection and its associated anaemia as evidenced in the current study could be a result of ASM in the study community, which requires expedited action to curtail the situation. Although our study concentrated only on malaria, ASM is also associated with several other health implications ranging from nutritional disorders to respiratory issues, renal diseases, poisoning, cardiovascular and gastrointestinal diseases, tuberculosis and pneumonia, among others [32]. Artisanal mining has been a cancer in most African countries, polluting water bodies and causing various disease conditions and other social problems, and the earlier that governmental and non-governmental institutions stand up against it, the better for the health and well-being of all.

\section{Other factors influencing malaria infection in ASM sites}

The results of this study revealed that children whose caregivers had good knowledge of malaria transmission and prevention had 30\% less risk of malaria infection than those with no information or poor knowledge. Some studies have attributed caregivers' educational level to children's risk of malaria infections, with children whose caregivers had no education at all having higher risk than those with higher education [14]. It could be assumed that caregivers with higher formal education are more likely to have greater awareness and knowledge of malaria infection, as found in the study by Mukomena et al. in Dembele kebele in south-eastern Ethiopia [33]. Our study, however, found that caregivers' educational level influenced malaria among their wards only at the bivariate level. Though it is unclear why this was not significant at the multivariate level, the generally low level of education throughout the ASM communities could be a factor.
The study also found that children under five with a history of frequent malaria had significantly greater odds of having malaria during the study period than those with no history of frequent malaria infection. This finding could be attributed to inadequate treatment of previous malaria infections by caregivers, resulting in children becoming asymptomatic carriers of infection. This assertion is further strengthened by the study findings, with a significant proportion of caregivers seeking malaria treatment from pharmacies, faith healers, spiritualists and herbalists or self-medicating rather than seeking care from a health centre. Previous studies by Nacher et al. [27] and Douine et al. [34] in French Guiana and the Amazon have shown similar findings.

Studies in Tanzania and Uganda found that older children were at greater risk of being infected with malaria compared to infants $[35,36]$. This could be explained by the fact that infants have immunity acquired from their mothers, including passive transfer of antibodies through breastfeeding. With increasing age, this immunity starts to wane, and hence children are at increased risk of malaria infection before they begin to develop their own immunity following repeated infections $[37,38]$. The present study, however, found no significant difference in malaria infections between children up to 11 months and those 12 months and older.

Despite caregiver knowledge and child history of malaria being significant factors in predicting malaria infection in this study, ASM remains the major source of exposure to infection and hence requires prudent measures to curb it.

\section{Limitations and strengths of the study}

Our study has a number of limitations. The first is the likelihood that participants may have brought a malaria infection from a different location due to travel. To ensure that our study findings were representative of the East Akim District, participants were selected based on having lived in the district for at least a month, which corresponds to the most frequent malaria incubation period with $P$. falciparum and the longest with $P$. malariae as indicated by the Centers for Disease Control and Prevention (CDC) [12].

Another drawback of this study is the possibility of incorrect malaria diagnosis among participants, as correct diagnosis requires technical expertise and skill. To mitigate this risk, quality control checks were performed on RDT test kits, and their results were accepted only when the control bands on the kits supported the interpretation of either a positive or negative test result. Additionally, the manufacturer's standard operating procedures were strongly adhered to. For malaria smear examinations, two certified and accredited microscopists 
who were blinded to the RDT results were engaged. A third microscopist was available in the case of divergent decisions in film/smear examination and reporting.

\section{Conclusions}

This study reveals a high prevalence of malaria parasite and infection (54.3\%) among children in artisanal mining communities of the East Akim District-a level much higher than that of the other districts, regions and the country at large. Not only is the prevalence high, but the asymptomatic (carrier) proportion is also alarming, with these infections being strongly associated with malaria. Stagnant residual water from mining activities in close proximity $(\leq 25 \mathrm{~m})$ to homes in these communities, inadequate control and experience of younger caregivers in handling the situation, and a child having a history of frequent malaria were factors associated with anaemia and malaria infection in this study. In light of the serious adverse effects associated with malaria among children, there is a pressing need to effectively regulate mining activities in resident communities, and to strengthen malaria control and education campaigns in these communities to reduce the high malaria prevalence and improve the health-seeking behaviour of community residents.

\begin{abstract}
Abbreviations
AOR: Adjusted odds ratio; CHPS: Community-based health planning services; $\mathrm{Cl}$ : Confidence intervals; COR: Crude odds ratio; FDA: Food and Drugs Authority; GHS: Ghana Health Service; m-RDT: Malaria rapid diagnostic test; NHIS: National Health Insurance Scheme; OPD: Outpatient department; OR: Odds ratio; RDT: Rapid diagnostic test; SD: Standard deviation; SHS: Senior high school; WHO: World Health Organization.
\end{abstract}

\section{Supplementary Information}

The online version contains supplementary material available at https://doi. org/10.1186/s13071-021-04919-8.

Additional file 1: Table S1. History of malaria-related signs/symptoms and caregivers' health-seeking behaviour for their child's symptoms including what action they took, whether they visited the health facility and the reasons for not visiting a health facility. Figures represent number and percentage (in parentheses). Table S2. Odds ratio of factors that influence malaria infections in children under five in East Akim district, Ghana.

\section{Acknowledgements}

We are grateful to WHO/TDR, the Office of Research Innovation and Development of the University of Ghana and the National Malaria Control Programme for supporting this study. We thank Dr Beni Prosper Tuosie, Municipal Health Directorate, East Akim District, Kibi; Mr Kwadwo Owusu Ansah and Ms Emmanuella Mensah Bonsu; and all the study participants. We are also thankful to all the research assistants, including Freda Asantewaa Manu, Bernice Clement, Owusua Yeboah Cynthia, Mavis Sintim, and Obed Oppong, who helped in collecting data from the study participants. We would also like to thank all who worked tirelessly to make the fieldwork in the study area possible, and the inhabitants of East Akim District.

\section{Authors' contributions}

The authors' contributions were as follows: conceptualization, FD and ADA; methodology, FD, GAA, SKD and ADA; formal analysis, FD, GAA and SKD; writing - original draft preparation, FD, SKD, CT, GAA, BS, KOA, PN and ADA; writing-review and editing, FD, SKD, CT, GAA, BS, KOA, PN and ADA; supervision of study, ADA. All authors read and approved the manuscript.

\section{Funding}

This study received financial assistance from the World Health Organization Special Programme for Research and Training in Tropical Diseases (WHO/ TDR). The funder had no role in the study design, data collection and analysis, manuscript preparation or decision to publish.

\section{Availability of data and materials}

The datasets used and/or analysed during the current study are available from the corresponding author on reasonable request.

\section{Declarations}

Ethics approval and consent to participate

A detailed plan of the study and its objectives were submitted to the ethical review committee of the Ghana Health Service (GHS) for approval, which was granted (Ghana Institutional Ethics Committee Approval number: GHS-ERC $137 / 12 / 17)$. The study was carried out in accordance with relevant guidelines and regulations. Permission was obtained from the East Akim Municipal Health Directorate for entry into the communities and health facilities. Informed consent was sought from caregivers and participants involved in the study. Participants were made aware that information obtained from them would only be used for improving malaria control activities in the district. Participants were also made aware that there were no known risks associated with their participation in the study, that participation was voluntary, and that they were free to withdraw from the study at any point. Access was limited to the principal investigator, two research assistants and the supervisor of the study. No identifying information such as patient name captured from the records was reported as part of the results.

\section{Consent for publication}

Not applicable.

\section{Competing interests}

The authors declare that they have no competing interests.

\section{Author details}

${ }^{1}$ Department of Epidemiology and Disease Control, School of Public Health, University of Ghana, Legon, Ghana. ${ }^{2}$ Malaria Research and Training Center, Department of Epidemiology and Infectious Diseases, University of Science Techniques and Technologies of Bamako, Bamako, Mali. ${ }^{3}$ National Malaria Control Programme, Accra, Ghana. ${ }^{4}$ Department of Optometry and Visual Science, College of Science, Kwame Nkrumah University of Science and Technology, Kumasi, Ghana. ${ }^{5}$ University of Ghana Centre for Evidence Synthesis and Policy, School of Public Health, University of Ghana, Legon, Ghana.

Received: 29 March 2021 Accepted: 2 August 2021

Published online: 21 August 2021

\section{References}

1. World Health Organization. World malaria report 2020: 20 years of global progress and challenges. 2020. https://www.who.int/docs/ default-source/malaria/world-malaria-reports/9789240015791-doublepage-view.pdf?sfvrsn=2c24349d_5. Accessed 19 Feb 2021.

2. World Health Organization. World malaria report 2019. 2019. https://apps. who.int/iris/bitstream/handle/10665/330011/9789241565721-eng.pdf. Accessed 20 Feb 2020.

3. World Health Organization. World malaria report 2018. 2018. http://apps. who.int/iris/bitstream/handle/10665/275867/9789241565653-eng.pdf. Accessed 22 May 2019. 
4. Programme GNMC. Annual report national malaria control programme 2015. 2015. https://www.ghanahealthservice.org/downloads/NMCP 2015_ANNUAL_REPORT.pdf. Accessed 20 Feb 2020.

5. National Malaria Control Programme UoHAS, AGA Malaria Control Programme, World Health Organization and the INFORM Project An epidemiological profile of malaria and its control in Ghana. A report prepared for the Ministry of Health, Ghana, the Roll Back Malaria Partnership and the Department for International Development, UK. November, 2013. https://web-archive.Ishtm.ac.uk/www.linkmalaria.org/sites/link/ files/content/country/profiles/Ghana-epi-report-2014.pdf. Accessed 20 Feb 2020.

6. Carneiro I, Roca-Feltrer A, Griffin JT, Smith L, Tanner M, Schellenberg JA, et al. Age-patterns of malaria vary with severity, transmission intensity and seasonality in sub-Saharan Africa: a systematic review and pooled analysis. PLOS ONE. 2010;5: e8988.

7. Programme GNMC. Annual bulletin 2016 national malaria control programme. 2016. https://ghanahealthservice.org/downloads/NMCP_ 2016\%20-Annual_Bulletin.pdf. Accessed 20 Feb 2020.

8. Ghana Statistical Service (GSS) GHSG, and ICF. Ghana malaria indicator survey 2016. 2017. https://dhsprogram.com/pubs/pdf/MIS26/MIS26.pdf. Accessed 20 Feb 2020.

9. Sanchez JF, Carnero AM, Rivera E, Rosales LA, Baldeviano GC, Asencios JL, et al. Unstable malaria transmission in the Southern Peruvian Amazon and its association with gold mining, Madre de Dios, 2001-2012. Am J Trop Med Hyg. 2017;96:304-11.

10. Valle D, Lima JM. Large-scale drivers of malaria and priority areas for prevention and control in the Brazilian Amazon region using a novel multi-pathogen geospatial model. Malar J. 2014;13:443.

11. Asante KP, Zandoh C, Dery DB, Brown C, Adjei G, Antwi-Dadzie Y, et al. Malaria epidemiology in the Ahafo area of Ghana. Malar J. 2011;10:211.

12. Prevention CfDCa. CDC - malaria—about malaria—disease. 2019 https://www.cdc.gov/malaria/about/disease.html. Accessed 20 Feb 2020.

13. GSS. 2010 Population and housing census. National analytical report. 2013. https://statsghana.gov.gh/gssmain/fileUpload/pressrelease/2010_ PHC_National_Analytical_Report.pdf. Accessed 20 Feb 2020.

14. Yankson R, Anto EA, Chipeta MG. Geostatistical analysis and mapping of malaria risk in children under 5 using point-referenced prevalence data in Ghana. Malar J. 2019;18:67.

15. Afolabi BM, Afolabi TM, Ogunwale A, Aiyesetenikan A. A 2-month intervention study of preventive clothing against mosquito bites among malnourished and well-nourished children under 5 years of age living on the Atlantic Ocean Coast of Lagos, Southwest Nigeria. Malar J. 2020;19:61

16. Wanzira H, Katamba H, Okullo AE, Agaba B, Kasule M, Rubahika D. Factors associated with malaria parasitaemia among children under 5 years in Uganda: a secondary data analysis of the 2014 malaria indicator survey dataset. Malar J. 2017;16:191.

17. Kabaghe AN, Chipeta MG, Terlouw DJ, McCann RS, van Vugt M, Grobusch MP, et al. Short-term changes in anemia and malaria parasite prevalence in children under 5 years during one year of repeated cross-sectional surveys in rural Malawi. Am J Trop Med Hyg. 2017;97:1568-75.

18. Nyarko SH, Cobblah A. Sociodemographic determinants of malaria among under-five children in Ghana. Malar Res Treat. 2014;2014: 304361.

19. da Silva-Nunes M, Moreno M, Conn JE, Gamboa D, Abeles S, Vinetz JM, et al. Amazonian malaria: asymptomatic human reservoirs, diagnostic challenges, environmentally driven changes in mosquito vector populations, and the mandate for sustainable control strategies. Acta Trop. 2012:121:281-91.

20. Knoblauch AM, Winkler MS, Archer C, Divall MJ, Owuor M, Yapo RM, et al. The epidemiology of malaria and anaemia in the Bonikro mining area, central Cote d'Ivoire. Malar J. 2014;13:194.

21. Oladeinde B, Omoregie R, Olley M, Anunibe J, Onifade A, Oladeinde O. Malaria and anemia among children in a low resource setting in Nigeria. Iran J Parasitol. 2012;7:31-7.
22. Eisele TP, Miller JM, Moonga HB, Hamainza B, Hutchinson P, Keating J. Malaria infection and anemia prevalence in Zambia's Luangwa district: an area of near-universal insecticide-treated mosquito net coverage. Am 」 Trop Med Hyg. 2011;84:152-7.

23. Pathak VA, Ghosh K. Erythropoiesis in malaria infections and factors modifying the erythropoietic response. Anemia. 2016;2016: 9310905.

24. Perkins DJ, Were T, Davenport GC, Kempaiah P, Hittner JB, Ong'echa JM. Severe malarial anemia: innate immunity and pathogenesis. Int J Biol Sci. 2011;7:1427-42.

25. Wegmuller R, Bentil H, Wirth JP, Petry N, Tanumihardjo SA, Allen L, et al. Anemia, micronutrient deficiencies, malaria, hemoglobinopathies and malnutrition in young children and non-pregnant women in Ghana: findings from a national survey. PLoS ONE. 2020;1 5: e0228258.

26. Shenton $L M$, Jones $A D$, Wilson ML. Factors associated with anemia status among children aged 6-59 months in Ghana, 2003-2014. Matern Child Health J. 2020;24:483-502.

27. Nacher M, Guerin PJ, Demar-Pierre M, Djossou F, Nosten F, Carme B. Made in Europe: will artemisinin resistance emerge in French Guiana? Malar J. 2013;12:152.

28. Amoah LE, Opong A, Ayanful-Torgby R, Abankwa J, Acquah FK. Prevalence of G6PD deficiency and Plasmodium falciparum parasites in asymptomatic school children living in southern Ghana. Malar J. 2016;15:388.

29. Crookston BT, Alder SC, Boakye I, Merrill RM, Amuasi JH, Porucznik CA, et al. Exploring the relationship between chronic undernutrition and asymptomatic malaria in Ghanaian children. Malar J. 2010;9:39.

30. Basu N, Clarke E, Green A, Calys-Tagoe B, Chan L, Dzodzomenyo M, et al. Integrated assessment of artisanal and small-scale gold mining in Ghana-part 1: human health review. Int J Environ Res Public Health. 2015;12:5143-76.

31. Hamza TAAN, Husen AA. Community knowledge, attitude and practice about malaria and mosquito biting behavior in southern Ethiopia. Am J BioSci. 2017;5:80-8.

32. Cossa $H$, Scheidegger R, Leuenberger A, Ammann P, Munguambe $K$, Utzinger J, et al. Health studies in the context of artisanal and small-scale mining: a scoping review. Int J Environ Res Public Health. 2021;18:1555.

33. Mukomena SE, Philipe CM, Desire MK, Pascal LT, Ali MM, Oscar LN. Asymptomatic parasitemia in under five, school age children and households self-medication, Lubumbashi, Democratic Republic of Congo. Pan Afr Med J. 2016;24:94.

34. Douine $M$, Lambert $Y$, Musset $L$, Hiwat $H$, Blume LR, Marchesini $P$, et al. Malaria in gold miners in the Guianas and the Amazon: current knowledge and challenges. Curr Trop Med Rep. 2020;7:37-47.

35. Hendriksen IC, White LI, Veenemans J, Mtove G, Woodrow C, Amos B, et al. Defining falciparum-malaria-attributable severe febrile illness in moderate-to-high transmission settings on the basis of plasma PfHRP2 concentration. J Infect Dis. 2013;207:351-61.

36. Ssempiira J, Nambuusi B, Kissa J, Agaba B, Makumbi F, Kasasa S, et al. Geostatistical modelling of malaria indicator survey data to assess the effects of interventions on the geographical distribution of malaria prevalence in children less than 5 years in Uganda. PLoS ONE. 2017;12: e0174948.

37. Doolan DL, Dobano C, Baird JK. Acquired immunity to malaria. Clin Microbiol Rev. 2009;22:13-36.

38. McLean ARD, Stanisic D, McGready R, Chotivanich K, Clapham C, Baiwog $F$, et al. P. falciparum infection and maternofetal antibody transfer in malaria-endemic settings of varying transmission. PLOS ONE. 2017;12: e0186577.

\section{Publisher's Note}

Springer Nature remains neutral with regard to jurisdictional claims in published maps and institutional affiliations. 